\title{
Los verdaderos problemas de la crisis de alimentos
}

Después de dos décadas de complacencia acerca de la alimentación, el mundo descubrió súbitamente en 1972 que aún no habia terminado de sepultar a Malthus. En Norteamérica, las grandes reservas de alimentos desaparecieron de pronto y los precios del trigo se multiplicaron con creces, de un nivel parejo de alrededor de 60 dólares la tonelada a más de 200 dólares, creando serios problemas a los países en desarrollo dependientes de la importación de productos alimenticios. Algunos países intentaron subsidiar los precios de los alimentos para proteger los salarios reales y casi llegaron a la quiebra, otros que no tenían la posibiliclad financiera para hacer esto, enfrentaron turbulencias sociales de intensidad variable. Pero entre aquellos más seriamente afectados, el hambre comenzó a amenazar la vida de millones. Aun en países occidentales, donde los gastos en alimentación constituyen una parte mucho más pequeña del presupuesto familiar, el alza de los precios de los alimentos ha contribuido a una desenfrenada inflación. Virtualmente, de la noche a la mañana, la alimentación se ha convertido en un serio problema mundial.

¿Por qué este repentino deterioro? ¿Se debía únicamente a malas concliciones climáticas o era sólo el comienzo de una crisis básica a largo plazo? Y, aún más importante, ¿cuál es la perspectiva alimenticia para los próximos 3, 5 ó 10 años? ¿Existe alguna posible solución para este problema?

Para responder a éstas y a otras preguntas relacionadas con éstas, las Naciones Unidas convocaron el año pasado a una conferencia especial sobre alimentación. La Conferencia tuvo lugar en Roma, a nivel ministerial, durante dos semanas en noviembre de 1974, y produjo, después de intensivos y calurosos debates, una impresionante declaración para la erradicación clel hambre y la desnutrición, y alrededor de 20 resoluciones acerca de temas que variaban desde "objetivos y estrategias de la producción de alimentos" hasta "mujer y alimentos". Este trabajo intenta evaluar los resultados de la Conferencia y discutir las implicaciones a largo plazo de la crisis alimenticia para los países en desarrollo. 


\section{LA EYALUAGIÓN}

La primera tarea de la Conferencia sobre Alimentos fue la de hacer una evaluación objetiva y autorizada de la situación presente y de las perspectivas de los alimentos, una identificación de su naturaleza y magnitud y luego obtener una base de àcuerdo para una acción nacional e internacional a fin de abordar el problema. La Conferencia cumplió esta tarea con notable claridad y con la casi total unanimidad.

La Conferencia estuvo de acuerdo con que la crisis a corto plazo fue causada por la contribución de factores circunstanciales, pero las causas de la crisis yacen mucho más profundas. El problema comenzó a surgir en 1972, cuando malas condiciones atmosféricas afectaron varios continentes, y la producción mundial de cereales de alrededor de 1.200 millones de toneladas descendió en 33 millones de toneladas, por primera vez descle la Segunda Guerra Mundial, en vez de aumentar en 25 millones de toneladas para alcanzar el aumento mínimo anual en la demanda mundial. Esta inesperada baja repentinamente agotó las reservas de los principales países exportadores y condujo a una aguda alza de los precios de los alimentos en los mercados del mundo. La cosecha de 1973 fue grande, pero no lo suficiente para prevenir un mayor agotamiento de las reservas. En 1974, el mal tiempo nuevamente afectó las cosechas en muchas partes del mundo y la producción total de cereales bajó en términos absolutos, por la segunda vez en tres años, pero sin el cojín de las reservas mundiales disponibles a principios de 1972. Esto ha agravado la crisis alimenticia y muchos lugares de Asia y Africa se enfrentan ahora con una seria escasez de alimentos. El número de muertes por desnutrición alcanza ya números de seis cifras. Muchos otros problemas tales como la inestabilidad monetaria, el problema de la energía, el miedo. a un receso mundial y una atmósfera general de incertidumbre acerca del futuro han ahondado las dificultades del sector agricola. La escasez de fertilizantes y sus altos precios se están transformando en un importante obstáculo en los esfuerzos para aumentar la producción, mientras las situación de las balanzas de pagos de muchos países en desarrollo han minado seriamente su capacidad para importar alimentos.

La Conferencia también estuvo de acuerdo en que la crisis alimenticia no había sido causada simplemente por razones de clima. Se estaba preparando desde hacía largo tiempo debido a profunda- 
mente enraizados factores seculares. El más importante de éstos era la inhabilidad de los países en desarrollo como grupo, para alcanzar su creciente demanda de alimentos. Entre 1952 y 1962, el aumento promedio anual de la producción alimenticia fue 3,I por ciento, considerablemente más alto que su promedio de crecimiento anual de población del 2,4 por ciento y casi al mismo nivel con el aumento total de la demanda de alimentos. Pero entre 1962 y 1972, la tasa de aumento de la producción alimenticia declinó levemente, de un promedio de 3,1 por ciento a un 2,6 por ciento anual, mientras el crecimientos anual de la demanda de alimentos subió de un promedio de 3,0 a 3,3 por ciento al año, principalmente por la aceleración de la tasa de aumento de la población en el último período. Como resultado, la brecha entre la producción y la demanda alimenticias comenzó a ensancharse gradual e imperceptiblemente. Después de haber sido exportadores netos de cereales antes de la Segunda Guerra Mundial, los países en desarrollo se han transformado en importadores sustanciales de alimentos, con importaciones anuales netas de 30 millones de toneladas para 1969-71, contra sólo \& millones de toneladas en 1950-52.

El segundo desequilibrio importante en la situación alimenticia mundial, como muchos delegados de la Conferencia hicieron notar, era su extremadamente mala distribución. Aún hoy habría suficiente comida para todos, pero los países en desarrollo están imposibilitados de adquirirla en los mercados, mientras los países desarrollados no estén dispuestos a proveer más granos en términos concesionales. Los países desarrollados, con alrededor del 30 por ciento de la población mundial, produjeron alrededor del 60 por ciento del total de granos, pero utilizaron casi el $60 \%$ de ellos (o más de 400 millones de toneladas al año) en la crianza del ganado: Sólo a través de esta política deliberada de subsidiar el uso de los granos para alimentación animal, junto a medidas tendientes a restringir la producción doméstica, los países desarrollados han conseguido eliminar el peso de los excedentes que habrían acumulado a lo largo de dos décadas y asegurar un mejor balance entre Ia demanda comercial mundial de alimentos y su oferta total. Pero estas políticas también condujeron a un modelo desafortunado de consumo, en el cual una parte del mundo tiene demasiados alimentos y la otra parte no los suficientes, sin un mecanismo aceptable para equilibrar o mejorar esta parte.

El tercer rasgo importante en la evaluación a largo plazo enfatizada por la Conferencia era el estado crónico de hambre y desnutrición en el mundo. De acuerdo con las últimas estimaciones de la FAO, alrededor de 460 millones de personas en Africa, Asia y América Latina, o casi el 20 por cicnto de su población, sufren de perpetua 
hambre y desnutrición. Esta situación sólo puede haberse agravado en estos dos años pasados. De algún modo, todos deben aprender a vivir con esta realidad trágica, pero ahora ha surgido como un grave problema y como la manifestación real del problema alimenticio a largo plazo.

Con respecto a las perspectivas futuras en materia de alimentos, la Conferencia convino en que "la situación tenía que agravarse considerablemente en el futuro, a no ser que se diera comienzo inmecliatamente a una acción apropiada".

De acuerdo con las proyecciones de la FAO cuidadosamente preparadas de antemano para la Conferencia, la demanda de alimentos en los próximos 10 años en los países en desarrollo debería aumentar en un 3,6 por ciento por año, contra un 3,3 por ciento en los años precedentes. Si el aumento en su producción alimenticia no se aceleraba sustancialmente sobre las tasas obtenidas en la última década (2,6 por ciento al año), entonces los requerimientos de importaciones alimenticias aumentarían de 30 millones de toneladas en 1969-7I a por lo menos 85 millones de toneladas para 1985, y posiblemente 100 millones de toneladas en años de malas cosechas. Esta se convertiría en una situación totalmente inmanejable mucho antes de que la brecha realmente alcanzara el nivel proyectado. De hecho, sólo basta con mirar los problemas creados por la actual brecha de alimentos de 7-8 millones de toneladas para apreciar ampliamente la naturaleza catastrófica de estas proyecciones.

Al aceptar esta evaluación de la situación alimenticia y al contemplar el estado crónico de desnutrición en el mundo, la conferencia expresó "seria preocupación de que el eterno problema del hambre, que ha perseguido hombres y naciones a través de la historia, haya adoptado ahora una importancia y urgencia sin precedentes y que sólo pueden enfrentarse con una acción mundial concertada".

\section{LAS SOLUGIONES}

El primer logro real de la Conferencia fue indudablemente el amplio interés y preocupación que generó acerca del hambre y la desnutrición, como un problema frontal que debe ser enfrentado y controlado internacionalmente, como las plagas -viruela y malaria- los otros enemigos comunes del hombre.

Segundo, la Conferencia aceptó la exterminación del hambre y la desnutrición, como el objetivo de la comunidad internacional como un todo y también el fin específico de que "dentro de una década 
ningún niño se acostará con hambre, ninguna familia temerá por el pan del día siguiente, y que el futuro y la capacidad de ningún ser humano se verán impedidos por la desnutrición." Estas palabras tomadas del discurso inaugural del doctor Henry Kissinger, el Secretario de Estado de los Estados Unidos, constituye ahora un solemne compromiso de toda la comunidad internacional, frente al cual sus políticas y acciones futuras deberán ser juzgadas.

Tercero, la Conferencia aceptó la necesidad de mayor ayuda alimenticia de emergencia y recomendó que "todos los países donantes acepten e implementen el concepto de continuar con los planes alimenticios y hacer todos los esfuerzos para proporcionar productos y/o asistencia financiera que pucliera asegurar en términos físicos al menos 10 millones de toneladas de granos como ayuda alimenticia anual, comenzanclo en 1975, y también para proveer una adecuada cantidad de otros productos alimenticios.

Cuarto, Ia Conferencia alcanzó un acuerdo en relación con un programa internacional de seguridad alimenticia mundial, basado en un sistema coordinado de mantención de reservas de cereales y apoyado en un sistema munclial de información alimenticia y de advertencias previas contra escaseces de alimentos, para garantizar que una alimentación adecuada está siempre clisponible y a precios razonables.

Quinto, y tal vez lo más importante, la Conferencia reafirmó que a fin de resolver el problema de los alimentos se debía dar la más alta prioridad a políticas y programas para aumentar la producción alimenticia en los países en desarrollo, de tal modo de alcanzar una tasa de crecimiento agrícola mínima de 4 por ciento al año. Adoptó algunas importantes resoluciones concernientes a fertilizantes, pesticidas, semillas, investigación, tierras, agua y desarrollo rural.

Sexto, reconociendo la necesidad de un aumento sustancial en la inversión agrícola, para obtener la aceleración requerida de al menos un cincuenta por ciento en el crecimiento promedio anual de la producción de alimentos en los últimos diez años (de 2,6 por ciento a 4 por ciento por año), la Conferencia resolvió establecer inmediatamente un fondo internacional para el desarrollo agrícola. Esta resolución fue propuesta por los principales países exportadores de petróleo y fue coauspiciada por 9 de los 13 miembros de la Organización de Países Exportaclores de Petróleo (OPEC).

Séptimo, la Conferencia dedicó gran canticlad de atención y tiempo a los arreglos institucionales necesarios para garantizar una adecuada ejecución de estas y otras clecisiones adoptadas por la Conferencia y acordó organizar un Consejo Alimenticio Mundial a nivel ministerial y plenipotenciario como un órgano de la Asamblea General de las Naciones Unidas, que funcionaría como un mecanismo 
de coordinación superior de políticas concernientes a la producción de alimentos, nutrición, seguridad y ayuda alimenticia, para implementar las resoluciones de la conferencia sobre estos temas.

Estos son logros sólidos, y hasta donde una conferencia internacional puede llegar, ésta puede ser considerada con el correr del tiempo como la más exitosa de las reuniones de la NU realizadas hasta ahora. Sin embargo, ¿pueden estas decisiones y recomendaciones en su conjunto realmente resolver el problema de alimentos? ¿Serán ellas implementadas total y efectivamente $y$ aun si lo son, verdaderamente abarcan todos los problemas fundlamentales?

LOS PROBLEMAS REALES

El problema de la alimentación visto objetivamente a la luz de las afirmaciones anteriormente resumidas puede parecer bastante comprensible, y las soluciones recomendadas practicables. Sin embargo, el problema tiene muchas fases políticas, sociales, económicas y morales, y cada una de ellas presenta sus propias complejidades y contradicciones.

A corto plazo, la posibilidad de salvar cientos de miles de personas de morir de hambre en los próximos doce meses parece bastante oscura. Y esto, de hecho, fue un gran fracaso de la Conferencia. Ya era conocido por los participantes el hecho de que 30 de los países más seriamente afectados necesitaban por lo menos 8 millones de toneladas de granos para alcanzar sus requerimientos mínimos de importación para el año 1974-75. Sus necesidades totales de importación para el año se estimaba en 16 millones de toneladas, de las cuales ya había arreglos para comprar 6 millones comercialmente y 2 millones por ayuda alimenticia. Pero no existía la probabilidad ni la garantía de que podrían recibir ayuda adicional o asistencia financiera para importar la cantidad restante. La perspectiva para 1975 , se ve nuevamente ensombrecida por una prolongada sequía en el subcontinente indio y en partes de Africa y por las dificultades de balanza de pagos, que afectan a la mayoría cle los países en clesarrollo más seriamente afectados.

Si la meta acordada de ayuda alimenticia de 10 millones de toneladas para los próximos tres años (comparada con el flujo anual de ayuda de alrededor de 5 millones de toneladas en los últimos dos años) puede ser rápidamente implementada, las perspectivas más inmediatas de inanición masiva pueden ser evitadas. Pero esto no será fácil, en parte por la resistencia de los gobiernos para otor- 
gar recursos presupuestarios adicionales para ayuda alimenticia, y en parte por la indeseable preocupación acerca de la oferta interna y de los precios de los alimentos en los países exportadores. Además, y ésta es la primera vez, la comunidad internacional ha aceptado una meta global de ayuda alimenticia en términos cuantitativos y el compartir esta meta entre los principales países donantes va a presentar muchos problemas. Esta ayuda no puede ser de exclusiva responsabilidad de los países exportadores de alimentos. Pero una gran parte de la ayuda alimenticia es también un componente de la asistencia total para el desarrollo. ¿Cómo se puede desarrollar un criterio aceptable para compartir tan sólo el objetivo de la ayuda alimenticia, particularmente entre los donantes tradicionales y los países exportadores de petróleo? También la meta de 10 millones fue aceptada por la Conferencia como una meta minima; sin embargo, las perspectivas de ir más lejos, a pesar de la necesidad desesperada de muchos países, no parece ser muy brillante en esta etapa. Tal vez una de las primeras tareas para el recién creado Consejo Mundial de Alimentación deberá ser, resolver estas controversias.y facilitar una pronta implementación de los objetivos de la ajuda alimenticia como el medio más rápido para enfrentar el problema de los alimentos a corto plazo.

A mediano plazo, por ejemplo, en los tres a cinco próximos años si el compromiso internacional para la seguridad alimenticia es implementado en forma efectiva y los principales países productores reúnen una provisión de alimentos, previniendo que la cosecha de los próximos dos o tres años sea los suficientemente abundante para ello, los precios de los alimentos pueden bajar. Esto, ayudado por el sistema propuesto de alarma anticipacla, mejoró los mecanismos para enfrentar emergencias, $y$ la política de ayuda alimenticia puede construir los cimientos de un sistema mundial de seguridad alimenticia. Sin embargo, no existe la certeza de que todos los principales elementos de tal sistema van a emerger en un futuro próximo.

El sistema de seguridad alimenticia, adoptado y recomendado por: la Conferencia, es mucho más modesto que el esquema que el extinto Lord Boyd Orr presentó a la Segunda Conferencia de FAO en 1946, para un consejo mundial sobre alimentación, que mantendría las reservas internacionales de alimentos para ayudar a los países necesitados y para estabilizar los precios de los alimentos. El concepto de seguridad alimenticia, según el compromiso internacional adoptado por la Conferencia, sólo proporciona "pautas internacionales para las políticas nacionales de reservas" y consultas periódicas acerca de la adecuación de dichas reservas. El primer paso deberia ser determinar un nivel "seguro" de reservas mundiales, sobre $y$ por encima de las reservas comerciales u operativas normales que deberán ser reunidas para permitir a los gobiernos y a los países exportadores e importadores 
participar en ellas sobre bases previamente acordadas. Aun cuando se logró un acuerdo preliminar sobre el objetivo global y sobre la participación inicial de los países, el funcionamiento del sistema podría plantear algunos problemas difíciles. Las reservas, una vez constituidas más allá de cierto punto, tienen un efecto depresivo en los precios y los países exportadores están naturalmente interesados en mantener las reservas a un nivel que no empuje los precios tan abajo como para debilitar los incentivos de los productores. ¿Por qué los países exportadores deberían constituir reservas mayores y aceptar precios más bajos, junto con soportar el alto costo de almacenamiento, a no ser que otros países estén dispuestos a compartir la carga? ¿Cómo pueden diversos países con objetivos variables ponerse de acuerdo sobre niveles mínimos de reservas y en márgenes de precios dentro de los cuales regular las reservas? Además, ningún exportador quiere hablar de estabilización de precios cuando éstos son elevados y están subiendo. Tendremos seguramente que esperar cosechas mayores y precios declinantes, antes de que el sistema aceptado por la Conferencia adquiera forma concreta.

La presente atmósfera política y económicamente desfavorable en el mundo sólo puede agravar estas dificultades para implementar las decisiones de la Conferencia. Muchos países están agobiados por sus propias dificultades internas y los peligros de una confrontación rápidamente están sobrepasando las oportunidades para una cooperación internacional fructífera. ¿Recibirá el problema alimenticio, como un problema humano básico, un tratamiento especial en esta atmósfera?

La solución a largo plazo del problema alimenticio, yace por supuesto, tanto en la reducción de la demanda como en el aumento de la producción, particularmente en los países en desarrollo.

En los países en desarrollo, el principal factor del incremento de la demanda de alimentos es el crecimiento de la población. Sin embargo, la población que deberá ser alimentada durante los próximos 10-15 años, ya ha nacido. En otras palabras, la demanda de alimentos en los países en desarrollo para el período hasta 1985 a aun 1990 , realmente no puede ser comprimida. Al mismo tiempo, sin una drástica reducción en la tasa de crecimiento de la población de ahora en adelante, el problema de alimentos más allá de 1990 se transformará en algo casi inmanejable.

Existen algunos analistas morbosos que inconscientemente predicen una reducción en la tasa de crecimiento de la población a través de una tasa de mortalidad mayor, debida principalmente al hambre y a la desnutrición. Pero si llevaran su análisis un poco más allá descubrirían que una reducción temporal de la población debida al hambre sería muy pronto seguida de una mayor tasa de crecimiento 
de la población. Estudios llevados a cabo en áreas seleccionadas afectadas por el hambre muestran claramente que una mayor proporción de gente vieja muere de inanición, aumentando así la proporción de la población en condiciones para tener hijos en el futuro. $Y$, en todo caso, cómo se pueden ignorar las consecuencias psicológicas, politicas y morales de tal "solución".

La demanda de granos en los países desarrollados puede, sin embargo, ser reducida, no en un sentido absoluto, pero disminuyendo la tasa de expansión en el uso de granos para el ganado y mediante una utilización más eficiente de otros alimentos. Teóricamente, aun un 2 por ciento de reducción en la alimentación total de granos del ganado en los países desarrollados en 1974, ahorraría grano suficiente para cubrir la brecha anual de 8 millones de toneladas en los países en desarrollo. Pero en la práctica, esta reducción no se obtendrá fácilmente y plantea algunos serios problemas para los países desarrollados. ¿Qué deben hacer si continúan produciendo más alimentos de los que necesitan? Los países en desarrollo que necesitarían este excedente de alimentos para el consumo humano pueden no tener los recursos para comprarlo comercialmente, mientras los países ricos pueden concebiblemente usar más y más granos para la alimentación animal. ¿Y cuánto grano, después de todo se puede esperar razonablemente que ellos distribuyan como ayuda, si existe demanda comercial de éste aun para alimentación animal?

Por lo tanto, la única solución fundamental a largo término del problema alimenticio yace en el aumento de la producción en los países en desarrollo, particularmente en los más pobres y populosos, que están en el centro del problema. Pero aquí, nuevamente, los factores desfavorables parecerían superar a los favorables.

Primero, la presión de la creciente demanda de alimentos, como muchos expertos han recalcado, está socavando ecológicamente algunos de los principales sistemas productores de alimentos del mundo. Existen problemas de pesca excesiva, sobreextensión de pastizales, desforestación, erosión del suelo, unidos a períodos y frecuencias anormales de sequías e inundaciones; expertos en climatología están también presintiendo pautas de deterioro climático.

Segundo, mientras nos volvemos forzosamente a los recursos marginales de tierra y agua, la economía de la producción agrícola ha cambiado drásticamente, en especial debido a los altos requerimientos de energía y capital de la tecnología existente, que hasta ahora se ha concentrado en una limitada variedad de cosechas. No hemos, hasta ahora, advertido las graves implicaciones de este cambio, porque los precios de los productos son relativamente altos. Pero el problema está justo a la vuelta de la esquina. Los precios a los cuales se pueden obtener incrementos adicionales de la producción, en paí- 
ses desarrollados y en desarrollo, utilizando recursos marginalés y aplicando dosis más altas de fertilizantes y pesticidas, no pueden ser soportados por los consumidores, particularmente en los países en desarrollo.

Tercero, los requerimientos de asistencia externa para inversiones en agricultura son mucho mayores que los que parecen ser posibles de movilizar bajo la presente atmósfera desfavorable. De acuerdo con estimaciones presentadas a la Conferencia, los objetivos mínimos propuestos de producción alimenticia no serán conseguidos a no ser que el flujo de recursos externos para el sector agrícola pueda ser aumentado del nivel actual de alrededor de 1.500 millones de dólares a, por lo menos, 5.000 millones de dólares al año, la mayoría en términos blandos. En el pasado, la agricultura no ha sido un sector elegible para la ayuda y ha recibido tan sólo el 5 . por ciento de la asistencia total para el desarrollo. Inclusive ésta ha ido principalmente a países que pueden afrontar préstamos en términos comerciales, puesto que los dos tercios de los recursos disponibles lo están sólo sobre bases comerciales. Es en este contexto que la resolución que establece un Fondo Internacional para el Desarrollo Agrícola podría tal vez ser considerada como el más importante resultado de la Conferencia sobre Alimentación. Pero hasta ahora no existen las garantías de que este fondo vaya a conducir realmente a un aumento sustancial de recursos en la escala requerida para la producción agrícola y alimenticia.

Peno los obstáculos más importantes para el aumento de la producción en los países en desarrollo son sociales y políticos. Auri si los programas propuestos apuntan hacia un aumento de la inyersión, proporcionando insumos y reforzando la investigación y se proporcionan otros servicios por sí mismos no podrían eliminar. la pobreza rural, abordar el problema del desempleo agrícola o proveer de alimentos suficientes a esa cuarta parte de la población que en los países en desarrollo está verdaderamente hambrienta y desnutrida. Lo esencial del problema alimenticio radica en producir y adoptar un modelo de desarrollo que comprende los segmentos más pobres de la población rural - el pequeño campesino y el trabajador sin tierra- en el proceso de producción y empleo. A no ser que y hasta que esto se. logre, los recursos, los insumos y la tecnologia van a resolver sólo una parte del problema, el alimento que se produzca no será distribuido equitativamente y la gente más pobre en cada uno de los países afectados permanecerá hambrienta.

El cómo comprometer a los pueblos no privilegiados en el proceso de desarrollo ha emergido tal vez como el problema más crítico sin resolver en la búsqueda de estrategias de desarrollo más apropiadas. Las respuestas son difíciles porque abarcan la escala total de las 
estructuras políticas de poder, tradiciones y actitudes sociales, modelos cle propiedad y' actividad económica y capacidades y limitaciones administrativas. Cacla país clebe lanzar su propia estrategia para el desarrollo que esté basada en su economía característica, en su situación social y ecológica, pero existen ciertos elementos fundamentales en el clesarrollo rural que son comunes y críticos. Estos incluyen el reconocimiento del elemento humano como el factor más importante en el proceso de clesarrollo, un marco institucional y político que movilice y organice los recursos humanos para aplicar una tecnología más avanzada en la agricultura, para diversificar las autoridades de la comunidad rural y para proporcionar servicios sociales básicos. La verdaclera prueba es el progreso en materia cle desarrollo rural, grande o pequeño, para trabajar en conjunto $y$ desarrollar sus recursos nacionales $y$ humanos para alcanzar sus crecientes necesidacles. Sin un proceso amplio y autososteniclo como este de desarrollo económico y social que ataque directamente la pobreza rural y urbana, la verclaclera solución al problema alimenticia no será encontrada.

Este descarnado análisis de algunos de los problemas reales en la crisis actual de alimentos no debe llevarnos a la conclusión de que el problema alimenticio se ha transformado en insoluble. De hecho, el problema es básicamente manejable pero requerirá un esfuerzo nacional tremendo en los países en clesarrollo y una cooperación sincera a nivel internacional.

DEL GREGIMIENTO A LA SUPERVIVENCIA

La crisis cle alimentos, en este análisis final, refleja el fracaso de los países en clesarrollo como grupo, para lograr sus objetivos de desarrollo económico y social en una escala adecuada. Estos objetivos se median hasta ahora en términos de crecimiento económico y cambios estructurales reflejados por el ritmo de la industrialización. Ahora ellos necesitan una nueva estrategia cle clesarrollo simplemente para sobrevivir. La perspectiva de una rápicla industrialización capaz de producir toda clase de bienes superfluos para el consumo masivo ya no resulta viable, incluso para los países desarrollados. Los países en clesarrollo no tienen ya otra opción sino la de trasladarse hacia una estrategia para el clesarrollo más austera $y$ autosostenicla, en la cual el énfasis principal será el clesarrollo de los recursos locales físicos y humanos para satisfacer las necesidacles básicas de alimentos, vestuario, habitación y medicina. 
Entretanto, la Conferencia sobre Alimentación les ha proporcionado un respiro de pocos años en los cuales hacer una propuesta importante para movilizar sus propios recursos y para aborclar el problema alimenticio. Además de proporcionar los comienzos de un sistema de seguridad alimenticia, la Conferencia puede conducir a un aumento en el flujo de recursos financieros, mejorar la provisión de importaciones tales como fertilizantes e intensificar un esfuerzo de investigación, pero el problema es mucho más profundo y su solución real se encuentra en los países en desarrollo. 